\title{
The effect of the juvenile hormone analog, fenoxycarb, on ecdysone receptor $B 1$ expression in the midgut of Bombyx mori during larval-pupal metamorphosis
}

\author{
Ebru Goncu, Osman Parlak \\ Department of Biology, Ege University, Faculty of Science, Turkey
}

\begin{abstract}
The Bombyx mori (Lepidoptera: Bombycidae) midgut undergoes remodeling during the larval-pupal metamorphosis. All metamorphic events in insects are controlled by mainly two hormones: 20-hydroxyecdysone (20E) and juvenile hormone (JH). Fenoxycarb, O-ethyl N-(2-(4-phenoxyphenoxy)-ethyl) carbamate, has been shown to be one of the most potent juvenile hormone analogs against a variety of insect species. In this study, the effect of fenoxycarb on EcR-B1 protein expression in the midgut of Bombyx mori during the remodeling process was investigated. Fenoxycarb was topically treated to the beginning of the fifth instar Bombyx larvae. Its application prolonged the last instar and prevented metamorphic events. Analyses were performed from day 6 of the fifth instar to $24 \mathrm{hr}$ after pupation in controls and to day 14 of the fifth instar in the fenoxycarb treated group. According to our results, the presence of EcR-B1 in the midguts of the fenoxycarb treated group during the feeding period suggested that EcR-B1 was involved in the functioning of larval cells and during this period fenoxycarb did not affect EcR-B1 status. Immediately after termination of the feeding stage, the amount of EcR-B1 protein increased, which indicated that it may strengthen the ecdysone signal for commitment of remodeling process. In the fenoxycarb treated group, its upregulation was delayed, which may be related to the inhibition of ecdysone secretion from the prothoracic gland. (Folia Histochemica et Cytobiologica 2012, Vol. 50, No. 1, 52-57)
\end{abstract}

Key words: Bombyx mori, ecdysone receptor B1, fenoxycarb, midgut

\section{Introduction}

Insect metamorphosis is controlled by mainly two hormones; 20 hydroxyecdysone $(20 \mathrm{E})$ and juvenile hormone $(\mathrm{JH})$. The first of these, $20 \mathrm{E}$, is a steroid hormone and its molecular actions are well known. During insect life, 20E initiates the molting process and regulates the gene expression needed for metamorphic changes by binding to a nuclear receptor which acts as a ligand dependent transcription factor [1]. Functional ecdysone receptors are heterodimers containing the ecdysone receptor and its partner ultraspiracle (USP) [2]. Three EcR isoforms (EcR-A, EcR-B1 and EcR-B2) were first identified in Drosophila melanogaster $[3,4]$ and the homologous of EcR-A

Correspondence address: E. Goncu, Department of Biology Ege University, Faculty of Science,

35100, Bornova - Izmir, Turkey;

e-mail: ebru.goncu@ege.edu.tr and EcR-B1 were detected in Manduca sexta [5] and Bombyx mori [6-8]. Different developmental profiles of receptor isoforms within the various tissues suggested that they are involved in stage specific hormonal responses $[4,9,10]$. For example, programmed cell death (PCD) in the anterior silk glands during larval pupal metamorphosis is triggered by ecdysone, and during this period it has been reported that there may be a connection between an increase in EcR-B1 protein and PCD process [11]. EcR-A was found in imaginal tissues of Drosophila melanogaster, while larval tissues abundantly express EcR-B1 [4].

$\mathrm{JH}$ is an acyclic sesquiterpenoid that prevents $20 \mathrm{E}$ induced gene expression. Unlike $20 \mathrm{E}$, the molecular actions of $\mathrm{JH}$ remain a mystery. Because of its unstable nature, juvenile hormone analogs are widely used in JH-related studies. Fenoxycarb, O-ethyl $\mathrm{N}$-(2-(4-phenoxyphenoxy)-ethyl) carbamate, is one of the most potent juvenile hormone analogs against a variety of insect species. The effects of fenoxycarb during 
the last larval instar in B. mori have been studied in detail [12-16]. Fenoxycarb application in the last larval instar delays, or completely inhibits, larval-pupal metamorphosis in a dose-dependent and application time-dependent manner [14]. Other juvenile hormone analogs methoprene [17] and ZR 515 [18] also show similar effects. Dedos and Fugo [15] reported that induction of dauer larvae by fenoxycarb in $B$. mori is related to the ecdysone secretion ability of the prothoracic glands, especially between $66 \mathrm{~h}$ and $72 \mathrm{~h}$ of the last instar.

The insect digestive system consists of three different regions: foregut, midgut and hindgut. The larval midgut is composed of a single layered epithelium containing columnar cells, goblet cells, regenerative (stem) cells and endocrine cells. In the prepupal stage, the midgut undergoes a remodeling process. Larval midgut epithelium degenerates via programmed cell death and the pupal midgut, which consists of thin and tall epithelial cells, is formed by the proliferation and differentiation of regenerative cells [19]. These metamorphic events are triggered by the steroid hormone ecdysone [20].

There is limited information about one of the ecdysone receptor isoforms (EcR-B1) protein level in the midgut of Bombyx mori [8] and there is no effect reported in the literature of the juvenile hormone analog, fenoxycarb, on its expression.

In this study, we aimed to analyze the effects of the juvenile hormone analog fenoxycarb on one of the ecdysone receptor isoforms EcR-B1 during the remodeling process of the midgut. $1 \mathrm{ng}$ of fenoxycarb treatment on day 0 delayed larval-pupal ecdysis and the fifth instar lasted 14 days. EcR-B1 was determined in the nucleus of larval midgut cells, both control and fenoxycarb treated groups. Its level increased after cessation of feeding, but then decreased before pupal ecdysis, and in addition EcR-B1 was not found in pupal midgut cells. Interestingly, EcR-B1 was found in the midguts of the fenoxycarb treated group during the feeding stage. These results suggest that EcR-B1 level may be important for larval midgut cells in response to $20 \mathrm{E}$ and during this period its expression was not affected by fenoxycarb, but its upregulation in the fenoxycarb treated group was inhibited until day 10 of the fifth instar. After day 11, fenoxycarb treated larvae showed wandering behavior which indicated that the prepupal period started on this day of the fifth instar. According to our previous study, larval midgut degeneration started on day 8 of the fifth instar in controls and day 12 in the fenoxycarb treated group [21]. The timing of the increase in EcR-B1 level just before the signs of programmed cell death during the early prepupal period (day 6,7 ) in the control group and day 11 in the fenoxycarb treated group may indicate that the midgut responds to ecdysone, especially for the commitment of the programmed cell death period, via this receptor isoform.

\section{Material and methods}

Animals. Japanese $\times$ Chinese hybrid races of the silkworm, Bombyx mori, were reared on fresh mulberry leaves at $25 \pm$ $\pm 1^{\circ} \mathrm{C}, 75-80 \%$ relative humidity and (LD 12:12) photoperiod conditions. Immediately after the fourth larval ecdysis, the silkworms were topically treated with fenoxycarb (1 ng/ $/ 10 \mu \mathrm{l})$ dissolved in acetone. Controls received only $10 \mu \mathrm{l}$ acetone. The final (fifth) larval instar lasts 10 days: seven days for feeding, followed by three days of spinning to build the cocoon in control groups. Experiments were performed from day 6 larvae of the fifth instar to $24 \mathrm{hr}$ after pupation in the control group; with the treatment group, they were carried out until the $14^{\text {th }}$ day of the fifth instar.

Immunohistochemistry. Midguts were dissected from day 6 larvae to $24 \mathrm{~h}$ after pupation in controls, and to day 14 of the fifth instar in the fenoxycarb treated group. Firstly, the silkworms were immobilized on ice. Each of the larvae was dissected from thorax to last abdominal segments. Midguts were separated gently, then washed with insect Ringer's solution (130 mM NaCl, $4.7 \mathrm{mM} \mathrm{KCl}, 1.9 \mathrm{mM} \mathrm{CaCl}_{2}$ ) to remove the content of the midgut and other body fluids. The dissected midguts were fixed immediately in Bouin's solution (water-saturated picric acid:formalin:acetic acid, 15:5:1 by volume). After fixation, tissue pieces were processed for embedding in paraffin wax. Five micrometer-thick sections were cut and mounted on lysine-coated slides. Sections were dewaxed in xylene for $15 \mathrm{~min}$, rehydrated through an ascending series of ethanol, then washed twice in phosphate-buffered saline (PBS) with $0.2 \%$ Tween 20 . Non-specific binding of the primary antibodies was blocked by incubating the sections with blocking solution (0.01 M PBS containing: $2 \%$ goat serum [Sigma, G9023], 1\% bovine serum albumin [BSA], $1 \%$ Triton X-100, $0.05 \%$ Tween $20,0.05 \%$ sodium azide, $\mathrm{pH} 7.2$ ) for $30 \mathrm{~min}$. Sections were then incubated at $4^{\circ} \mathrm{C}$ overnight with monoclonal EcR-B1 antibody (6B7, Developmental Studies Hybridoma Bank, University of Iowa, Iowa City, IO, USA) at a 1:1,000 dilution. To quench endogeneous peroxidase activity, sections were then incubated with $3 \% \mathrm{H}_{2} \mathrm{O}_{2}$ for $10 \mathrm{~min}$. They were then incubated in horseradish peroxidase-conjugated secondary antibody (Genetex, GTX85313) at a 1:200 dilution for $2 \mathrm{~h}$. Visualization was performed by incubation with the substrate and chromogen, 3',3'diaminobenzidine (DAB, Sigma-Aldrich D5905). Negative controls were performed by replacing the primary antibody with normal goat serum (Sigma, G9023) at the same dilution. The sections were examined under an Olympus BX-51 microscope and photographed with an Olympus digital camera. In order to assess labeling, sec- 
tions were scored from 1 to 3 according to intensity of labeling: 1 = weak labeling; 2 = moderate labeling; 3 = intense labeling.

Western blotting. Midguts were homogenized in $20 \mathrm{mM}$ Tris$-\mathrm{HCl}$ ( $\mathrm{pH} 7.5$ ) containing protease inhibitor cocktail (Roche) and $0.5 \%$ Nonidet P-40. The homogenate was centrifuged at $16,000 \times \mathrm{g}$ for $10 \mathrm{~min}$ at $4^{\circ} \mathrm{C}$. Total protein concentration was calculated with the BCA protein assay kit (Pierce). Twenty $\mu \mathrm{g}$ of total proteins were subjected to $10 \%$ SDS-polyacrylamide gel electrophoresis in a gel running buffer (25 mM Tris, 192 mM Glycine, 0.1\% SDS, pH 8.3) using Bio-Rad vertical electrophoresis. Proteins were electrotransferred onto a nitrocellulose membrane using Bio-Rad Transblot cell. Membranes were placed in blocking solution (50 mM Tris- $\mathrm{HCl}, \mathrm{pH} 7.5,150 \mathrm{mM} \mathrm{NaCl}, 1$ mM EDTA, and $0.1 \%$ Tween 20 (TBST) containing $1 \%$ bovine serum albumin and $5 \%$ dried non-fat milk overnight at $4{ }^{\circ} \mathrm{C}$. They were then incubated with 6B7 anti EcR-B1 monoclonal antibody [22] in TBST (1:700) for $2 \mathrm{~h}$ followed by $2 \mathrm{~h}$ incubation with horseradish peroxidase conjugated secondary antibody (Genetex, GTX85313). Detection was performed by chemiluminescence (Pierce, ECL Western blotting substrate, 32106) according to the manufacturer's instructions. Blots were scanned (Hp Psc 1315) and relative abundance of protein was calculated using ImageJ software from the National Institutes of Health, USA (http://rsb.info.nih.gov/ /nih-image/).

\section{Results}

Control group larvae ceased feeding activity at the end of day 7 and gut purge occurred on this day. Larvae started spinning behavior on day 8 , and $97 \%$ of larvae showed larval-pupal ecdysis on day 11 . The length of the last larval stage was prolonged by about four days in the fenoxycarb treated group. Larvae ceased feeding on day 11 and spinning was seen on day 12 . In this group, $92 \%$ of larvae showed spinning behavior. Following gut purge, remodeling process in midgut began in both the control and the treatment group. Larval midgut epithelium degenerated via programmed cell death and pupal midgut was formed by regenerative cells. In the control group, EcR-B1 was exhibited in larval midgut by using immunohistochemical method and Western blotting. Labeling intensity was moderate on day 6 (scored 2.1) in the control. After cessation of feeding, an increase in intensity of EcR-B1 immunolabeling of nuclei on days 7 and 8 was observed on paraffin sections (scored 3.1 and 2.8 respectively). After this day, immunolabeling become weaker day by day until pupation. EcR-B1 immunoreactivity was not determined in pupal midgut of the control group (Figures 1, 2A). The calculated relative abundance of protein suggested that the amount of EcR-B1 protein increased at feeding termination (day 7), and this level was not maintained during the prepupal period in control (Figure 2B). EcR-B1 level began to decrease before larval-pupal ecdysis. The last larval period was extended in the fenoxycarb treatment group and remodeling processes of midgut were delayed like other metamorphic changes. Nuclear EcR-B1 immunoreactivity persisted in the fenoxycarb treated group during the experiment period. Weak labeling intensities were detected on the first four days of the experiment (scored 0.7 to 0.9 ). After day 9, the immunoreactivity of EcR-B1 became intense, and moderate labeling was observed on day 10 . The strongest immunosignals were detected on days 11 and 12 (scored 2.4 and 2.6, respectively) then decreased sharply on days 13 and 14 (scored 0.8 and 0.4 respectively). Results from Western blot experiments indicate that a relatively low amount of EcR-B1 protein was detected until day 10 of the fifth instar (Figures 3, 4A). In this group, EcR-B1 was upregulated after cessation of feeding on day 11 of the fifth instar, then decreased over the last two days of the experiment (days 13 and 14) which was considered to be theprepupal period.

\section{Discussion}

The steroid hormone ecdysone triggers the remodeling period of midgut. $1 \mathrm{ng}$ of fenoxycarb treatment on day 0 of the fifth instar delayed midgut remodeling. Parthasaraty and Palli [23] reported that the application of another juvenile hormone analog methoprene to Heliothis virescens caused a delay or inhibition of midgut remodeling in dose-dependent manner. EcR-B1 was detected in the larval midgut of Bombyx mori during the feeding stage and just after termination of feeding, which suggested that EcR-B1 may be important for both function of larval midgut cell and stimulatory action of ecdysone for PCD. According to immunohistochemical analysis, labeling intensity was moderate on day 6 in the control group. After cessation of feeding, an increase in intensity of EcR-B1 immunolabeling of nuclei on days 7 and 8 was observed. Immunolabeling became weaker on days 9 and 10. Unlike the prepupal stage, EcR-B1 immunoreactivity was not determined in pupal midgut of control group (Figure 1). The relative abundance of EcR-B1 on each day was found to be consistent with the immunohistochemical results (Figure 3). Immunolocalization of EcR-B1 in midguts of fenoxycarb treated group was found to be different from the control group. Weak labeling intensities were detected on the first four days of the experiment on day 6 , day 7, day 8 and day 9. After day 9, the immunoreactivity of EcR-B1 became intense and moderate labeling was 

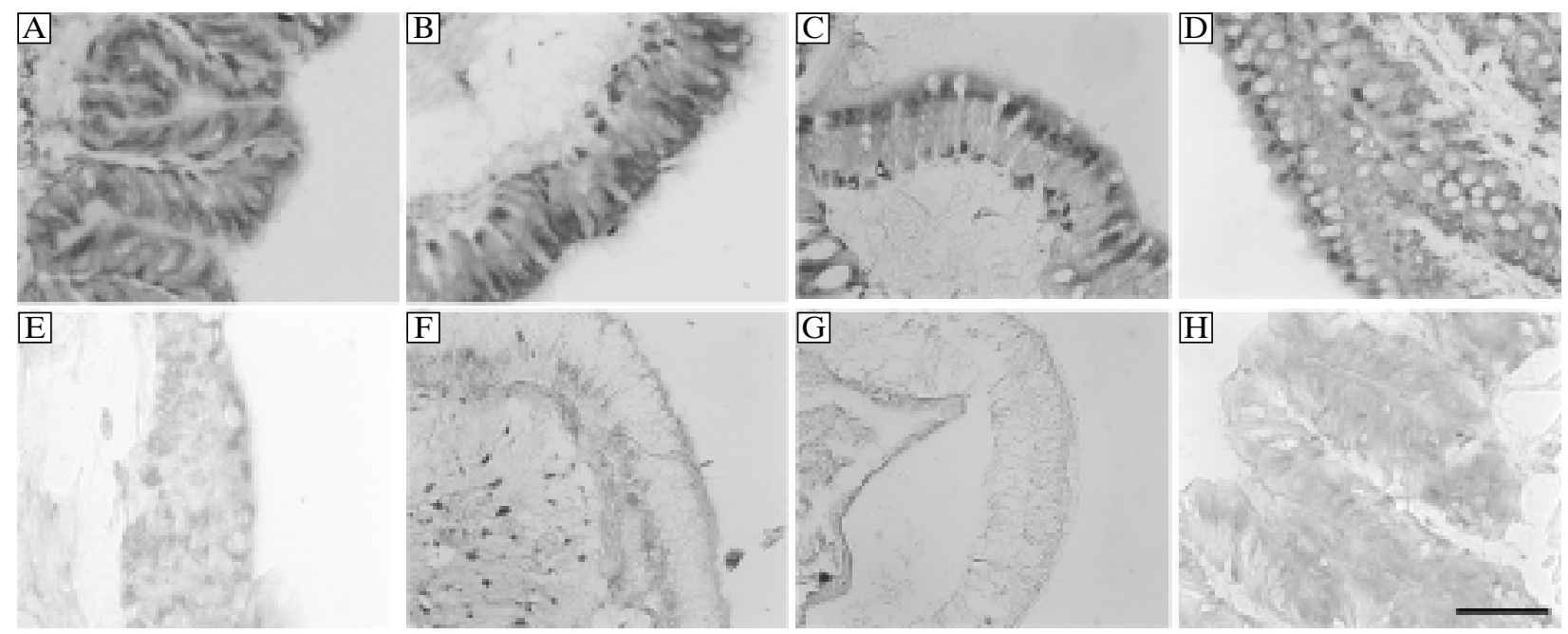

Figure 1. Demonstration of EcR-B1 in larval midguts of control group using immunohistochemical method. (A) day 6 , (B) day 7, (C) day 8, (D) day 9, (E) day 10, (F) at pupation, (G) 12 hours after pupation, (H) negative control midgut on day 7. Scale bar $150 \mu \mathrm{m}$ for $\mathrm{A}-\mathrm{G} ; 75 \mu \mathrm{m}$ for $\mathrm{H}$
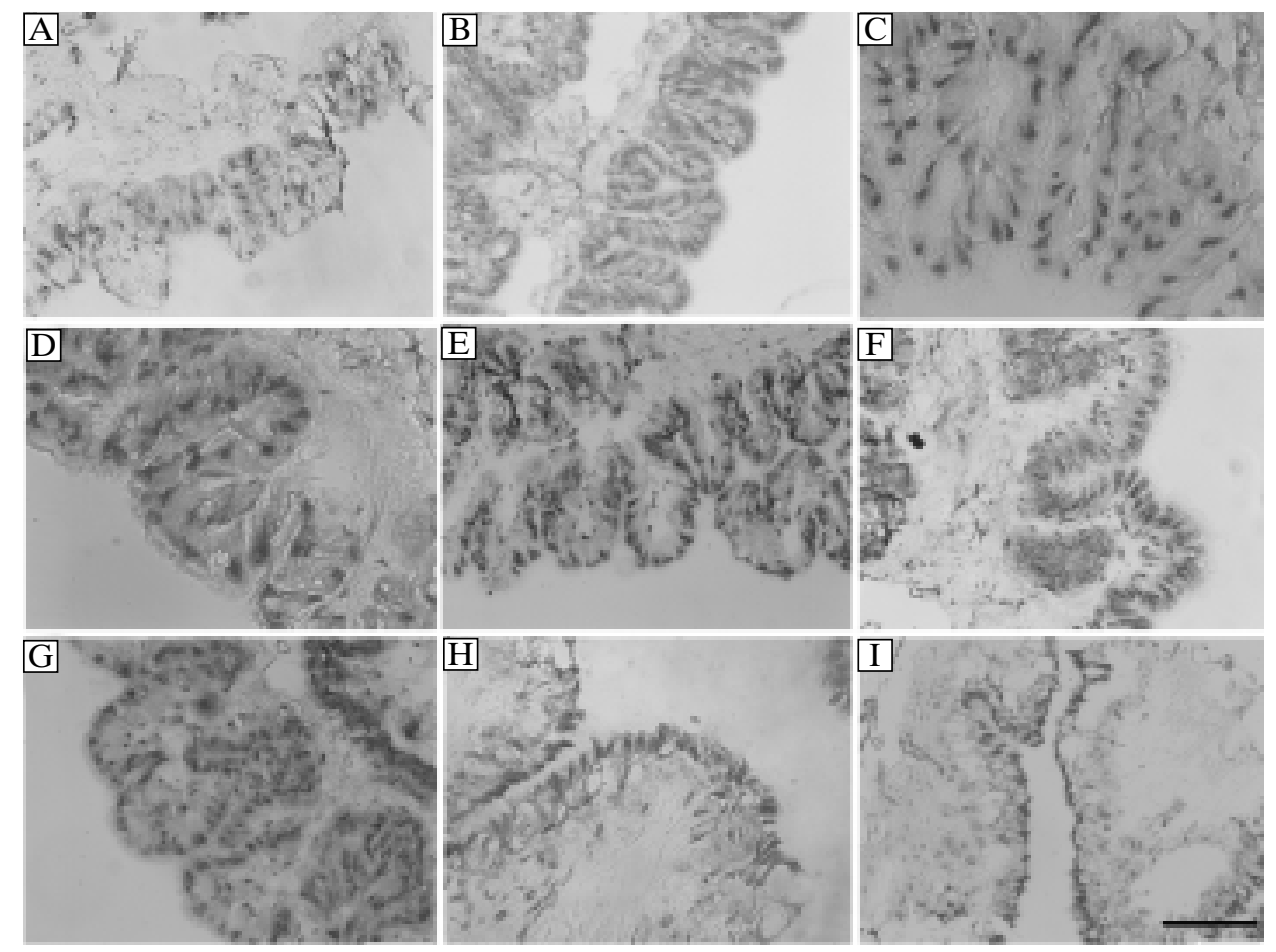

Figure 2. Immunolocalization of EcR-B1 in midguts of fenoxycarb treated group. (A) day 6, (B) day 7, (C) day 8, (D) day 9, (E) day 10, (F) day 11, (G) day 12, (H) day 13 and (J) day 14. Scale bar $150 \mu \mathrm{m}$ for B, E, F, G, H and J; $75 \mu \mathrm{m}$ for A, C and D

observed on day 10. The strongest immunosignals were detected on day 11, and then decreased sharply on days 13 and 14 (Figure 2). Western blotting showed similar results with immunohistochemistry (Figure 4).

Similar results have been found in the midguts of various different species. It has been reported that EcR-B1 mRNA increased in the midgut of Tribolium casteneum (Coleoptera: Tenebrionidae) just before pupation, but it was also reported that EcR-A expres- sion was involved in the regulation of EcR-B1 expression [24]. A rising EcR-B1 amount was detected in the midgut of Aedes aegyptii (Diptera: Culicidae) just before larval-pupal ecdysis, and its expression decreased below detectable levels after pupation [25]. Wu et al. [26] reported that EcR-B1 mRNA was involved during the midgut remodeling process of A. aegyptii and that methoprene treatment reduced EcR-B1 expression during pharate pupal stage but its ex- 
A
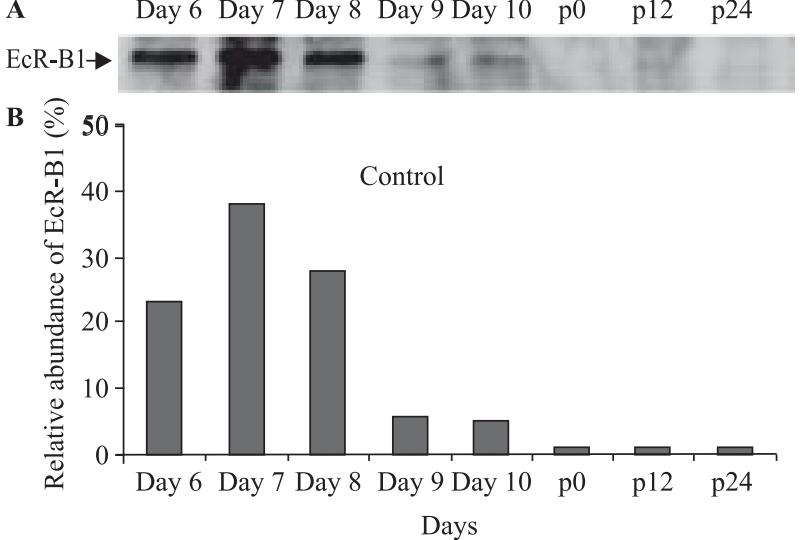

Figure 3. (A) Detection of EcR-B1 protein by Western blotting during remodeling process of midgut of Bombyx mori in control group. p0, at pupation; p12, $12 \mathrm{~h}$ pupa; p24, $24 \mathrm{~h}$ pupa, arrow indicates EcR-B1. (B) The average intensities of each band and relative abundance of each protein $(\mathrm{n}=7)$

A Day 6 Day 7 Day 8 Day 9 Day 10 Day 11 Day 12 Day 13 Day 14

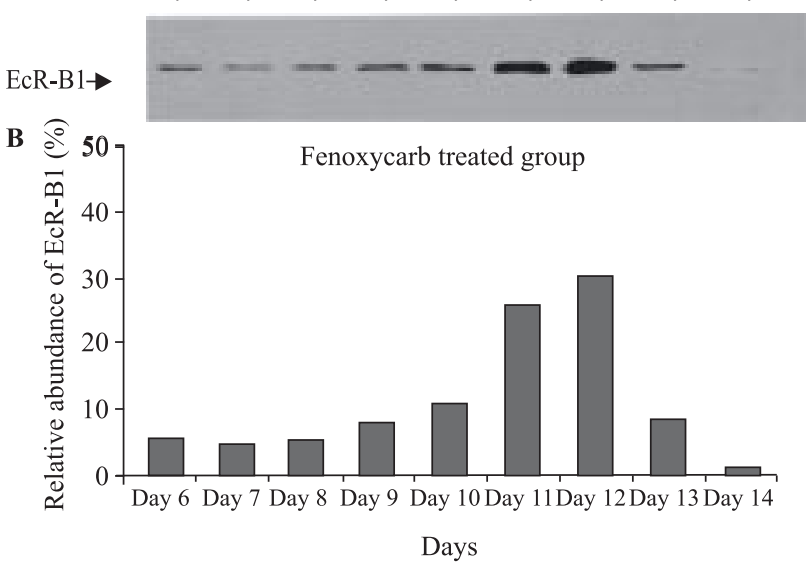

Figure 4. (A) Detection of EcR-B1 protein by Western blotting in midguts of fenoxycarb treated group.

(B) The average intensities of each band and relative abundance of each protein $(n=7)$

pression persisted during pupal period. According to our results, fenoxycarb application at the beginning of the fifth instar prevented upregulation of EcR-B1 on days 7 and 8 but its steady low level was maintained until day 11. Riddiford et al. [2] reported that ecdysone upregulates its own receptor. Therefore prevention of EcR-B1 upregulation may be caused by inhibition of ecdysone secretion from prothoracic gland which occurs on day 6 under physiological conditions [27]. The presence of EcR-B1 in the midguts of the fenoxycarb treated group during the feeding period suggested that EcR-B1 was involved in functioning of larval cells and during this period fenoxycarb did not affect its expression. The upregulation of EcR-B1 immediately after termination of the feeding stage may indicate that it may strengthen the ecdysone signal for commitment of the remodeling process.

\section{References}

1. Riddiford LM. Hormone action at the cellular level. In: Kerkut GA and Gilbert LI (eds.). Comprehensive Insect Physiology, Biochemistry and Pharmacology. Pergamon Press, Oxford 1985;37-84.

2. Riddiford LM, Hiruma K, Lan Q, Zhou B. Regulation and role of nuclear receptors during larval molting and metamorphosis of Lepidoptera. Am Zool. 1999;39:736-746.

3. Koelle MR, Talbot WS, Segraves WA, Bender MT, Cherbas P, Hogness DS. The Drosophila EcR gene encodes an ecdysone receptor, a new member of the steroid receptor superfamily. Cell. 1991;67:59-77.

4. Talbot WS, Swyryd EA, Hogness DS. Drosophila tissues with different metamorphic responses to ecdysone express different ecdysone receptor isoforms. Cell. 1993;73:1323-1337.

5. Fujiwara H, Jindra M, Newitt R et al. Cloning of an ecdysone receptor homolog from Manduca sexta and the developmental profile of its mRNA in wings. Insect Biochem Mol Biol. 1995;25:845-856.

6. Swevers L, Cherbas L, Cherbas P, Iatrou K. Bombyx EcR (BmEcR) and Bombyx USP (BmCF1) combine to form a functional ecdysone receptor. Insect Biochem Mol Biol. 1996;26:217-221.

7. Kamimura M, TomitaS, Fujiwara H. Molecular cloning of an ecdysone receptor (B1isoform) homologue from the silkworm, Bombyx mori, and its mRNA expression during wing disc development. Comp Biochem Physiol B Biochem Mol Biol. 1996;113:341-347.

8. Kamimura M, Tomita S, Kiuchi M, Fujiwara H. Tissue-specific and stage-specific expression of two silkworm ecdysone receptor isoforms. Ecdysteroid-dependent transcription in cultured anterior silk glands. Eur J Biochem. 1997;248:786-793.

9. Robinow S, Draizen TA, Truman JW. Genes that induce apoptosis: transcriptional regulation in identified, doomed neurons of the Drosophila CNS. Dev Biol. 1997;190:206-213.

10. Truman JW, Thorn RS, Robinow S. Programmed neuronal death in insect development.J Neurobiol. 1992;23:1295-1311.

11. Goncu E, Parlak O. Morphological changes and patterns of ecdysone receptor $\mathrm{B} 1$ immunolocalization in the anterior silk gland undergoing programmed cell death in the silkworm, Bombyx mori. Acta Histochem. 2009;111:25-34.

12. Kamimura M. Effects of juvenile hormone analogue, fenoxycarb, on larval growth of the silkworm, Bombyx mori (Lepidoptera: Bombycidae). Appl Entomol Zool. 1995;30:487-489.

13. Leonardi MG, Cappellozza S, Ianne P, Cappellozza L, Parentis P, Giordina B. Effects of the topical application an insect growth regulator (Fenoxycarb) on some physiological parameters in the fifth instar larvae of the silkworm Bombyx mori. Comp Biochem Physiol. 1996;113B:361-365.

14. Kamimura M, Kiuchi M. Effects of a juvenile hormone ana$\log$, fenoxycarb, on $5^{\text {th }}$ stadium of the larvae of the silkworm, Bombyx mori (Lepidoptera:Bombycidae). Appl Entomol Zool. 1998;33:333-338.

15. Dedos SG, Fugo H. Induction of dauer larvae by application of fenoxycarb early in the 5 th instar of the silkworm, Bombyx mori. J Insect Physýol. 1999;45:769-775.

16. Monconduit H, Mauchamp B. Fenoxycarb, a potent contaminant of the silkworm Bombyx mori L., does not influence its juvenile hormone titer. Arch Insect Býochem. 1999;40:141-149.

17. Miranda JE, Bortoli SA, Takahashi R. Development and silk production by silkworm larvae after topical application of methoprene. Sci Agr. 2002;59:585-588. 
18. Daillie J. Juvenile hormone modifies larvae and silk gland development in Bombyx mori. Biochimie. 1979;61:275-281.

19. Lehane M, Billingsley P. Structural biology of the midgut. In: Lehane ML, Billingsley PF (eds.). Biology of the Insect Midgut. Springer 1996:3-25.

20. Wigglesworth VB. Digestion and Nutrition: The principles of insect physiology. Chapman \& Hall, London 1972.

21. Goncu E, Parlak O. The influence of juvenile hormone analogue, fenoxycarb on the midgut remodeling in Bombyx mori (Lepidoptera: Bombycidae) during larval-pupal metamorphosis. Turkish Journal of Entomology. 2011; TED-2010$-026(35-2)$, in press.

22. Jindra M, Malone F, Hiruma K, Riddiford LM. Developmental profiles and ecdysteroid regulation of the mRNAs for two ecdysone receptor isoforms in the epidermis and wings of the tobacco hornworm, Manduca sexta. Dev Biol. 1996;180:258-272.

23. Parthasarathy P, Palli SR. Developmental and hormonal regulation of midgut remodeling in a Lepidopteran insect $\mathrm{He}$ liothis virescens, Mechanism of Development. 2007;124:23-34.
24. Tan A, Palli SR. Edysone receptor isoforms play distinct roles in controlling molting and metamorphosis in the red flour beetle, Tribolium castaneum. Mol Cell Endocrinol. 2008; 291:42-49.

25. Parthasarathy R, Palli SR. Stage- and cell-specific expression of ecdysone receptors and ecdysone-induced transcription factors during midgut remodeling in the yellow fever mosquito, Aedes aegypti. J Insect Physiol. 2007;53:216-229.

26. Wu Y, Parthasarathy R, Bai H, Palli SR. Mechanisms of midgut remodeling: juvenile hormone analog methoprene blocks midgut metamorphosis by modulating ecdysone action. Mechanisms of Development. 2006;123:530-547.

27. Sakurai S, Kaya M, Satake SI. Hemolymph ecdysteroid titer and ecdysteroid-dependent developmental events in the las larval stadium of the silkworm, Bombyx mori: role of low ecdysteroid titer in larval-pupal metamorphosis and a reappraisal of the head critical period. J Insect Physiol. 1998;44:867-881.

Submitted: 29 November, 2010

Accepted after reviews: 14 November, 2011 